\title{
Application value of expansive pedicle screw in the lumbar short-segment fixation and fusion for osteoporosis patients
}

\author{
FENGBIAO WENG* ${ }^{*}$ JIAZI WANG* ${ }^{*}$ LIWEN YANG, JINCAI ZENG, YAWEI CHU and ZHIGANG TIAN \\ Department of Orthopaedics, The First People's Hospital of Wujiang District, Suzhou, Jiangsu 215200, P.R. China
}

Received November 16, 2017; Accepted May 22, 2018

DOI: $10.3892 /$ etm.2018.6248

\begin{abstract}
Clinical value of expansive pedicle screw in lumbar short-segment fixation and fusion for patients with osteoporosis was investigated. A total of 80 patients with lumbar compression fracture but without obvious nerve compression were selected and divided into the observation group $(n=40)$ and the control group $(n=40)$ using a random number table. The observation group used the expansive pedicle screw, and the control group received conventional pedicle screw fixation and bone graft fusion. In the observation group, the operation and hospitalization time after operation were shorter and the intraoperative bleeding amount was less than that in control group $(\mathrm{p}<0.05)$. At 1 week, 1,3 and 6 months after operation, the observation group had better straight leg raising test (SLRT) scores, higher lower limb sensory scores but lower visual analogue scale (VAS) scores than control group $(\mathrm{p}<0.05)$. Besides, the proportions of postoperative infection, dural mater tear, nerve root injury and spinal cord injury during operation in the observation group were lower than those in the control group $(\mathrm{p}<0.05)$, and the bone graft fusion rates at 3 and 6 months after operation were obviously superior to those in control group $(\mathrm{p}<0.05)$. Moreover, after operation, the spinal stenosis rate in the observation group was lower than that in control group $(\mathrm{p}<0.05)$, the vertebral height ratio was larger than that in control group $(\mathrm{p}<0.05)$, and the Cobb's angle was smaller than that in the control group $(\mathrm{p}<0.05)$. In addition, there was a negative correlation between bone mineral density (BMD) and hospitalization time after operation in the observation group $(\mathrm{p}<0.05)$. In conclusion, the internal fixation with expansive pedicle screw for osteoporosis patients with lumbar compression fracture is characterized by short operation time, less intraoperative bleeding, few
\end{abstract}

Correspondence to: Dr Zhigang Tian, Department of Orthopaedics, The First People's Hospital of Wujiang District, 169 Park Road, Wujiang, Suzhou, Jiangsu 215200, P.R. China

E-mail: leoinwujiang@qq.com

*Contributed equally

Key words: expansive pedicle screw, osteoporosis, lumbar compression fracture, neurological function complications, quick recovery of postoperative neurological function and satisfactory surgical effect. However, reasonable intervention in osteoporosis is also necessary.

\section{Introduction}

Osteoporosis affects the spine most frequently and it is the most common in thoracolumbar spine, which often occurs in the elderly female patients. Once the osteoporosis occurs, it will seriously affect the bearing capacity and stability of spine, thus leading to spontaneous fractures (1). In the treatment of such patients with internal screw fixation, the strength of internal fixator and postoperative recovery will be negatively affected, which is the relevant risk factor to the postoperative complications and even surgical failure (2). Studies have shown that the proportion of postoperative screw loosening in patients complicated with osteoporosis is $\sim 15 \%$ (3). In the past, the internal fixation strength in osteoporosis patients with lumbar fracture was often enhanced through increasing the screw length and diameter, or combined application with bone cement (4), which, however, increased the risk of nerve and vascular injury, and even pedicle fracture (5). Expansive pedicle screw has higher internal fixation stability, especially anti-prolapse capacity, compared with the ordinary screw, so it has been paid increasingly more attention in clinical practice (6). In this study, the internal fixation treatment of lumbar short-segment compression fracture in osteoporosis patients with expansive pedicle screw was studied.

\section{Patients and methods}

General patient data. A total of 80 patients with lumbar compression fracture treated in the First People's Hospital of Wujiang District (Suzhou, China) from November 2015 to January 2017 were selected. All patients signed the informed consent before enrollment. This study was approved by the Ethics Committee of the First People's Hospital of Wujiang District.

Inclusion criteria: patients aged 45-70 years were definitely diagnosed with lumbar compression fracture and osteoporosis via clinical manifestations, computed tomography (CT), magnetic resonance imaging (MRI) examination and bone mineral density (BMD).

Exclusion criteria: patients with obvious nerve compression, coagulation dysfunction, mental illness, systemic 
infection, severe cardiopulmonary insufficiency or hepatorenal insufficiency, malignant tumor, fracture of lower limb, or low back pain caused by other reasons were excluded.

All patients enrolled were divided into the observation group $(n=40)$ and the control group $(n=40)$ using a random number table. In the observation group, there were 12 males and 28 females aged $45-70$ years $(65.5 \pm 2.0$ years on average) with the course of disease of 1-5 months $(3.0 \pm 0.3$ months on average). The nerve injury was classified according to the Frankel score: there were 11 cases of grade C, 23 cases of grade $\mathrm{D}$ and 6 cases of grade $\mathrm{E}$. In terms of the pathogenic site, there were 30 cases in $\mathrm{L}_{1}, 5$ cases in $\mathrm{L}_{2}$ and 5 cases in $\mathrm{L}_{3}$ and below. The anterior height ratio of injured vertebra was $25.0-75.0 \%(45.0 \pm 2.0 \%$ on average). The Cobb's angle was $15.0-40.5^{\circ}\left(29.3 \pm 1.1^{\circ}\right.$ on average). In the control group, there were 11 males and 29 females aged 45-70 years (65.6 \pm 2.0 years on average) with the course of disease of $1-5$ months ( $3.1 \pm 0.3$ months on average). The nerve injury was classified according to the Frankel score: there were 12 cases of grade C, 23 cases of grade D and 5 cases of grade E. In terms of the pathogenic site, there were 31 cases in $\mathrm{L}_{1}, 4$ cases in $\mathrm{L}_{2}$ and 5 cases in $\mathrm{L}_{3}$ and below. The anterior height ratio of injured vertebra was $25.0-75.0 \%(45.3 \pm 2.0 \%$ on average). The Cobb's angle was $15.0-40.5^{\circ}\left(29.5 \pm 1.0^{\circ}\right.$ on average). The sex, age, course of disease, Frankel score of nerve injury, pathogenic site, anterior height ratio of injured vertebra and Cobb's angle had no statistically significant differences between the two groups ( $\mathrm{p}>0.05)$.

Methods. All patients received the operative treatment via trachea cannula under general anesthesia. Under the prone position, patients with compression fracture underwent the manual reduction after successful anesthesia from posterior approach. Whether the total laminectomy and bone graft fusion were performed was determined based on the injury severity. The internal fixator used in the observation group was the expansive pedicle screw. The vertebral pedicle was drilled and the expansive pedicle screw was placed in it under the $\mathrm{C}$-arm guidance. A fine needle with $3 \mathrm{~mm}$ in diameter penetrated into the vertebral body for positioning and guidance, and then $\sim 2 \mathrm{ml}$ bone cement prepared in advance was injected using the injector into the hole on each vertebral pedicle. But the amount of bone cement was reduced appropriately for patients complicated with severe vertebral compression fracture, so as to reduce the bone cement leakage. After injection of bone cement, the syringe needle was immediately drawn out and the expansive pedicle screw was screwed into the vertebral pedicle along the hole approach. Then the expansive inner core was placed into the pedicle screw to fully expand the screw precursor, followed by fixation using screw rod. The tail cap was screwed and the autogenous bone cage was implanted. The control group was treated with conventional pedicle screw and bone graft fusion via the same surgical approach, as well as the screw placement and bone graft fusion under the $\mathrm{C}$-arm guidance.

Observational indexes. The operation time, intraoperative bleeding and postoperative indwelling drainage were compared between the two groups. After operation, the straight leg raising test (SLRT) score, visual analogue scale (VAS) score of lumbocrural pain and lower limb sensory score were evaluated in the postoperative follow-up. Moreover, the operation-related complications, the bone graft fusion rates at 3 and 6 months after operation, the spinal stenosis rates after operation, the vertebral height ratios and Cobb's angles of the two groups were recorded. Finally, the correlation between BMD and hospitalization time after operation in the observation group was analyzed.

Evaluation criteria. Sensory score: the puncture sensation and light touching of 17 dermatomes in bilateral lower limbs were scored. The total score is 112 point, and the higher the score is, the more perfect the function will be; the VAS score was used for lumbocrural pain: 10 points indicate unbearable pain, while 0 point indicates no pain. SLRT score: under the supine position on hard bed, the patients raised the leg on the affected side. Raising angle $\geq 70^{\circ}$ indicates normal, 0 point; $50-60^{\circ}$ for 1 point; $40-50^{\circ}$ for 2 points; $20-40^{\circ}$ for 3 points; $<20^{\circ}$ for 4 points; the higher the score is, the more obvious the clinical symptoms will be. Bridwell bone healing standard was used for bone graft fusion: level I: complete fusion and remodeling after intervention with newly-formed trabeculae; level II: complete bone block after intervention, but incomplete remodeling without lucent area; level III: complete bone block after intervention, but incomplete remodeling with a few lucent areas; level IV: significant collapse and absorption of bone block after intervention, no bone healing or remodeling. In this study, level I and II was included into the statistics of bone graft fusion. Diagnostic criteria of osteoporosis: it was mainly based on BMD, combined with the characteristics of Chinese population; generally, $\mathrm{L}_{3} \mathrm{BMD}$ lower than the average BMD for two standard deviations indicates osteoporosis; reference value of $\mathrm{L}_{3} \mathrm{BMD} \mathrm{SD}=1.228 \mathrm{~g} / \mathrm{cm}^{3}$; spinal stenosis rate $=[$ normal sagittal diameter $(\mathrm{A})-$ sagittal diameter in the narrowest part (B)]/A x100\%; vertebral height ratio (\%) is the geometric mean of adjacent vertebral height; Cobb's angle: it is measured in the adem position using the Cobb's angle protractor under the $\mathrm{X}$-ray guidance.

Statistical analysis. Statistical Product and Service Solutions (SPSS) 13.0 (SPSS, Inc., Chicago, IL, USA) was used for statistical treatment. Measurement data were presented as mean \pm standard deviation (SD), t-test was used for the comparison of means between the two groups, and the Chi-square test was used for the comparison of ratio between the two groups. $\mathrm{P}<0.05$ suggested that the difference was statistically significant.

\section{Results}

Comparison of the SLRT score, VAS score of lumbocrural pain, lower limb sensory score before operation between the two groups. The SLRT score, VAS score of lumbocrural pain and lower limb sensory score had no statistically significant differences before operation between the two groups, respectively $(\mathrm{p}>0.05)$.

Comparison of operation time, intraoperative bleeding and hospitalization time after operation between the two groups. In the observation group, the operation time was shorter than that in the control group $(\mathrm{p}<0.05)$, the intraoperative bleeding was less than that in the control group $(\mathrm{p}<0.05)$, and the 
Table I. Comparison of operation time, intraoperative bleeding and hospitalization time after operation between the two groups (mean \pm standard deviation).

\begin{tabular}{lccc}
\hline Groups & $\begin{array}{c}\text { Operation } \\
\text { time (min) }\end{array}$ & $\begin{array}{c}\text { Intraoperative } \\
\text { bleeding (ml) }\end{array}$ & $\begin{array}{c}\text { Hospitalization } \\
\text { time after } \\
\text { operation (days) }\end{array}$ \\
\hline $\begin{array}{l}\text { Observation } \\
\text { group }\end{array}$ & $149.7 \pm 8.7$ & $500.0 \pm 60.0$ & $12.6 \pm 2.3$ \\
Control & & & \\
group & $178.1 \pm 10.8$ & $1,000.0 \pm 100.0$ & $20.6 \pm 3.5$ \\
t & 12.952 & 27.116 & 12.081 \\
P-value & $<0.001$ & $<0.001$ & $<0.001$ \\
\hline
\end{tabular}

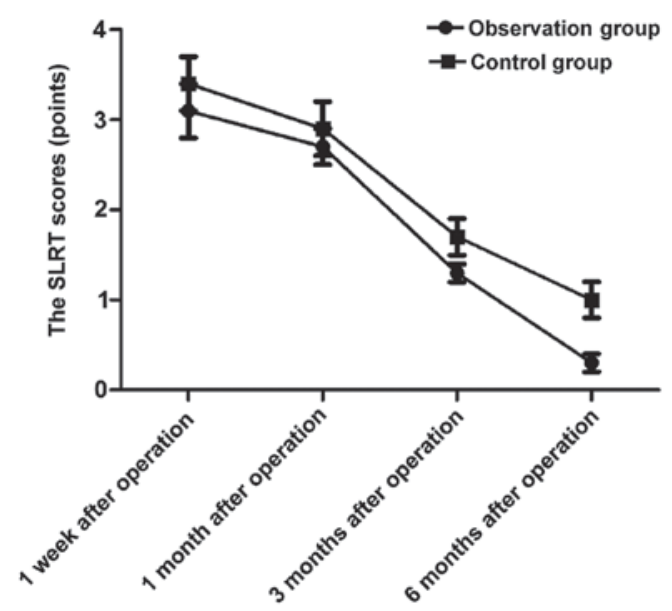

Figure 1. Comparison of SLRT scores in postoperative follow-up between the two groups. At 1 week, 1, 3 and 6 months after operation, the SLRT scores of the observation group are significantly superior to those of the control group $(\mathrm{p}<0.05)$. SLRT, straight leg raising test.

hospitalization time after operation was also shorter than that in the control group $(\mathrm{p}<0.05)$ (Table I).

Comparison of SLRT scores in postoperative follow-up between the two groups. At 1 week, 1, 3 and 6 months after operation, the SLRT scores of the observation group were $3.4 \pm 0.3,2.7 \pm 0.2,1.3 \pm 0.1$ and $0.3 \pm 0.1$ points, respectively, which were significantly superior to those of the control group $(3.4 \pm 0.3,2.9 \pm 0.3,1.7 \pm 0.2$ and $1.0 \pm 0.2$ points) $(\mathrm{t}=4.472,3.508$, 11.314 and 19.799; p<0.05) (Fig. 1).

Comparison of VAS scores of lumbocrural pain in postoperative follow-up between the two groups. At 1 week, 1 , 3 and 6 months after operation, the VAS scores of lumbocrural pain of the observation group were $5.3 \pm 0.4,3.4 \pm 0.2$, $2.3 \pm 0.2$ and $1.8 \pm 0.1$ points, respectively, which were significantly lower than those of the control group $(6.7 \pm 0.6,4.6 \pm 0.5$, $3.5 \pm 0.3$ and $2.6 \pm 0.2$ points) $(\mathrm{t}=12.279,14.093,21.049$ and 22.627; p<0.05) (Fig. 2).

Comparison of lower limb sensory scores in postoperative follow-up between the two groups. At 1 week, 1 , 3 and 6 months after operation, the lower limb sensory scores

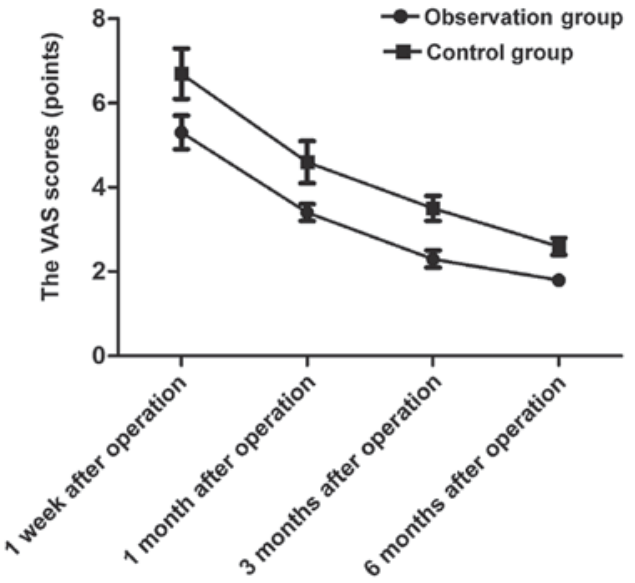

Figure 2. Comparison of VAS scores of lumbocrural pain in postoperative follow-up between the two groups. At 1 week, 1, 3 and 6 months after operation, the VAS scores of lumbocrural pain of the observation group are significantly lower than those of the control group $(\mathrm{p}<0.05)$. VAS, visual analogue scale.

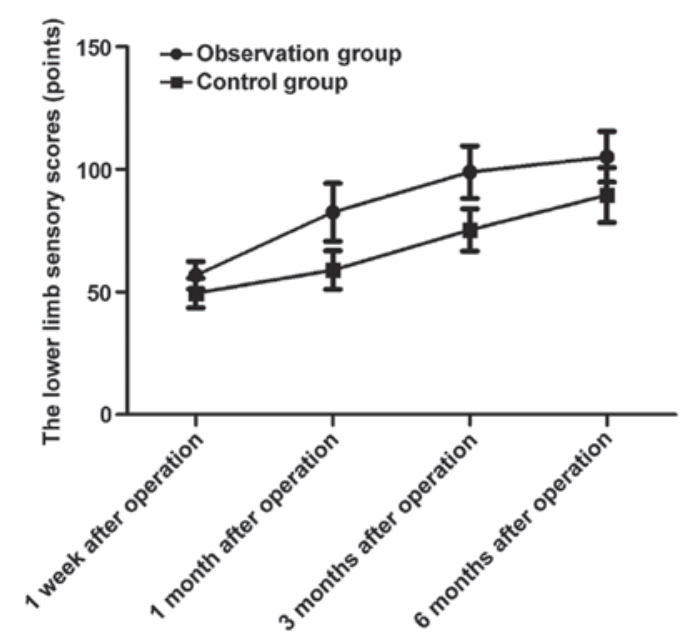

Figure 3. Comparison of lower limb sensory scores in postoperative follow-up between the two groups. At 1 week, 1, 3 and 6 months after operation, the lower limb sensory scores of the observation group are significantly higher than those of the control group $(\mathrm{p}<0.05)$.

of the observation group were $56.8 \pm 5.6,82.5 \pm 11.8,98.9 \pm 10.7$ and $105.1 \pm 10.3$ points, respectively, which were significantly higher than those of the control group $(49.6 \pm 6.0,58.9 \pm 7.9$, $75.3 \pm 8.6$ and $89.5 \pm 11.2$ points) $(\mathrm{t}=5.548,10.511,10.873$ and 6.484; p<0.05) (Fig. 3).

Comparison of operation-related complications between the two groups. The proportions of postoperative infection, dural mater tear, nerve root injury and spinal cord injury during operation in the observation group were lower than those in the control group $(\mathrm{p}<0.05)$ (Table II).

Comparison of bone graft fusion rates at 3 and 6 months after operation between the two groups. The bone graft fusion rates at 3 and 6 months after operation were obviously superior to those in the control group $(\mathrm{p}<0.05)$ (Table III).

Comparison of spinal stenosis rate, vertebral height ratio and Cobb's angle between the two groups. After operation, 
Table II. Comparison of operation-related complications between the two groups [n (\%)].

\begin{tabular}{lccccc}
\hline & $\begin{array}{c}\text { Spinal } \\
\text { cord } \\
\text { injury }\end{array}$ & $\begin{array}{c}\text { Dural } \\
\text { mater } \\
\text { tear }\end{array}$ & $\begin{array}{c}\text { Nerve } \\
\text { root } \\
\text { injury }\end{array}$ & $\begin{array}{c}\text { Wound } \\
\text { infection }\end{array}$ & $\begin{array}{c}\text { Total } \\
\text { incidence } \\
\text { rate }(\%)\end{array}$ \\
\hline $\begin{array}{l}\text { Observation } \\
\text { group }\end{array}$ & 0 & 1 & 0 & 2 & $3(7.5 \%)$ \\
$\begin{array}{l}\text { Control } \\
\text { group }\end{array}$ & 1 & 6 & 2 & 3 & $12(30.0 \%)$ \\
$\chi^{2}$ & & & & & 5.251 \\
P-value & & & & & 0.022 \\
\hline
\end{tabular}

Table III. Comparison of bone graft fusion rates at 3 and 6 months after operation between the two groups [n (\%)].

\begin{tabular}{lcc}
\hline Groups & $\begin{array}{c}3 \text { months } \\
\text { after operation }\end{array}$ & $\begin{array}{c}6 \text { months } \\
\text { after operation }\end{array}$ \\
\hline Observation group & $23(57.5 \%)$ & $36(90.0 \%)$ \\
Control group & $11(27.5 \%)$ & $20(50.0 \%)$ \\
$\chi^{2}$ & 7.366 & 15.238 \\
P-value & 0.007 & $<0.001$ \\
\hline
\end{tabular}

Table IV. Comparison of spinal stenosis rate, vertebral height ratio and Cobb's angle between the two groups (mean \pm standard deviation)

\begin{tabular}{lccc}
\hline Groups & $\begin{array}{c}\text { Spinal stenosis } \\
\text { rate }(\%)\end{array}$ & $\begin{array}{c}\text { Vertebral height } \\
\text { ratio }(\%)\end{array}$ & $\begin{array}{c}\text { Cobb's } \\
\text { angle }\left({ }^{\circ}\right)\end{array}$ \\
\hline Observation group & $3.6 \pm 0.2$ & $96.5 \pm 2.1$ & $3.0 \pm 0.2$ \\
Control group & $10.9 \pm 1.1$ & $86.5 \pm 3.3$ & $8.2 \pm 1.1$ \\
t & 41.295 & 16.169 & 29.416 \\
P-value & $<0.001$ & $<0.001$ & $<0.001$ \\
\hline
\end{tabular}

the spinal stenosis rate in the observation group was lower than that in the control group $(\mathrm{p}<0.05)$, the vertebral height ratio was larger than that in the control group $(\mathrm{p}<0.05)$, and the Cobb's angle was smaller than that in the control group $(\mathrm{p}<0.05)$ (Table IV).

Correlation analysis of BMD with hospitalization time after operation in the observation group. Pearson's correlation analysis showed that there was a negative correlation between BMD and hospitalization time after operation in the observation group [r=-0.9087; $\mathrm{p}<0.001(<0.05)]$ (Fig. 4).

\section{Discussion}

The pedicle screw fixation treatment is one of the good methods for the clinical treatment of lumbar compression fractures (7). However, the stability of conventional pedicle screw
Hospitalization time after operation (days) $[r=-0.9087 ; p<0.001(<0.05)]$

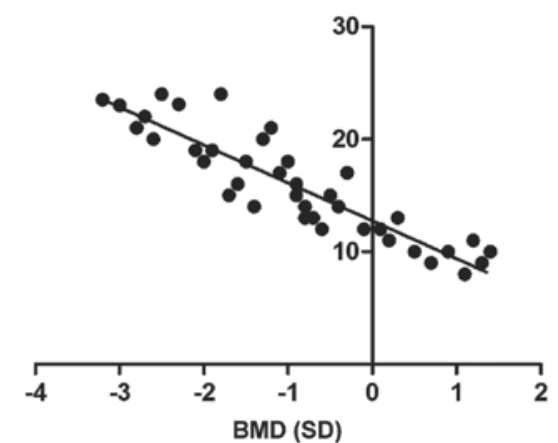

Figure 4. Pearson's correlation analysis of BMD with hospitalization time after operation in the observation group. There is a negative correlation between BMD and hospitalization time after operation in the observation group [r=-0.9087; $\mathrm{p}<0.001(<0.05)]$. BMD, bone mineral density.

fixation for patients with osteoporosis is significantly reduced due to lumbar osteoporosis, and it is difficult to achieve a solid internal fixation effect (8). Expansive pedicle screw can fix the vertical axial section through the front expansive effect (9), thus forming the triangular support (10), and significantly increasing the screw bonding (11); at the same time, the surrounding bone trabecula is appropriately compressed, thereby further improving the bone density and the stability of internal fixation (12). In addition, the front end of internal fixation screw after expansion can expand to both sides, thus effectively embedding into the bone and better improving the anti-rotation capacity of the screw (13). Therefore, expansive pedicle screw has been widely used in the treatment of lumbar compression fractures in patients with osteoporosis (14).

In this study, the observation group was treated with expansive pedicle screw, while the control group was treated with conventional internal screw fixation. In the observation group, the operation time was shorter than that in the control group, the intraoperative bleeding was less than that in the control group and the postoperative hospitalization time was shorter than that in the control group, suggesting that the expansive pedicle screw treatment of osteoporosis patients with lumbar compression fractures can significantly shorten the operation time, reduce the intraoperative bleeding and promote the postoperative recovery of patients. In addition, the SLRT score, VAS score of lumbocrural pain and lower limb sensory score were compared between the two groups in the postoperative follow-up and it was found that at 1 week, 1, 3 and 6 months after operation, the SLRT scores of the observation group were significantly superior to those of the control group, the VAS scores of lumbocrural pain were significantly lower than those of the control group, but the lower limb sensory scores were significantly higher than those of the control group, indicating that after the expansive pedicle screw treatment of osteoporosis patients with lumbar compression fractures, the lower limb motor and sensory functions of patients after operation are improved significantly, and the pain degree is reduced. At the same time, the study on the operation-related complications in the two groups showed that the proportions of postoperative infection, dural mater tear, nerve root injury and spinal cord injury during operation in the observation group 
were lower than those in the control group, and there were no spinal cord injury, nerve root injury or other severe peripheral nerve injury in the observation group, suggesting that the expansive pedicle screw treatment of osteoporosis patients with lumbar compression fractures has fewer postoperative complications than conventional internal screw fixation; in particular, it avoids the impact on the spinal cord, nerve root and other important tissues, so its safety is better. The bone graft fusion rates at 3 and 6 months after operation were obviously superior to those in the control group. Moreover, after operation, the spinal stenosis rate in the observation group was lower than that in the control group, the vertebral height ratio was larger than that in the control group, and the Cobb's angle was smaller than that in the control group, further suggesting that the expansive pedicle screw treatment of osteoporosis patients with lumbar compression fractures has a higher bone graft fusion rate, effectively improves the acute compression fractures, thus improving the surgical effects. Finally, the correlation analysis of BMD and hospitalization time after operation in the observation group showed that there was a negative correlation between BMD and hospitalization time after operation, indicating that osteoporosis has a negative effect not only on the conventional screw internal fixation treatment, but also on the expansive pedicle screw internal fixation treatment. Therefore, the expansive pedicle screw treatment of osteoporosis patients with lumbar compression fractures is not a universal method, and the strict control over indications and the active treatment of osteoporosis are of great significance in improving the prognosis of patients. These results are in accordance with previous studies $(15,16)$.

Expansive pedicle screw, compared with the conventional pedicle screw, mainly consists of two major components, hollow outer tube screws and built-in cylinder screw expander (17). During operation, the cylindrical tube screw is compressed to expand the hollow outer tube, making the diameter of nail tip reach $\sim 2.5 \mathrm{~cm}$ (18), thereby strengthening the compression against the osteoporotic vertebral bone around the nail and improving the screw bonding (19). One third of the end of expansive pedicle screw has no expansion effect (20), so the pedicle fracture is effectively avoided and the surgical results are improved; at the same time, the vertical tail of pedicle screw can realize the universal fixation (21), effectively avoiding the compression and enhancement during the conventional screw implantation, thereby shortening the operation time. In addition, after the screw implantation, the vertebral trabeculae around the implantation has a certain expansion and compression with the extension of implantation time (22), inducing longitudinal endogenous fusion and realizing the bone-nail fusion (23), which further increases the stability of screw fixation and reduces the risk of postoperative screw shedding and loosening. It must be mentioned, however, that a limitation of this study was the small sample size. Further studies with a larger number of samples will be needed in the future.

In conclusion, the internal fixation with expansive pedicle screw for osteoporosis patients with lumbar compression fracture is characterized by short operation time, less intraoperative bleeding, few complications, quick recovery of postoperative neurological function and satisfactory surgical effect. However, reasonable intervention in osteoporosis is also necessary.

\section{Acknowledgements}

Not applicable.

\section{Funding}

We received no funding support for this study.

\section{Availability of data and materials}

All data generated or analyzed during this study are included in this published article.

\section{Authors' contributions}

FW and ZT designed the study. JW, LY and JZ collected the data. FW and YC performed the data analysis and statistics. JW and LY conducted the data interpretation. FW prepared the manuscript. FW, JW and ZT performed the operations. All authors read and approved the final manuscript.

\section{Ethics approval and consent to participate}

This study was approved by the Ethics Committee of the First People's Hospital of Wujiang District (Suzhou, China). Signed informed consents were obtained from the patients or the guardians.

\section{Patient consent for publication}

Patients or their guardians provided written informed consents for publication.

\section{Competing interests}

The authors declare no competing interests.

\section{References}

1. Aycan MF, Tolunay T, Demir T, Yaman ME and Usta Y: Pullout performance comparison of novel expandable pedicle screw with expandable poly-ether-ether-ketone shells and cement-augmented pedicle screws. Proc Inst Mech Eng H 231: 169-175, 2017.

2. Kehayov II, Zhelyazkov CB, Kalnev BM, Davarski AN, Kitov BD and Raykov SD: Initial experience with $\mathrm{O}$-arm navigated spinal surgery - report on two cases. Folia Med (Plovdiv) 58: 293-298, 2016.

3. Wang MY, Chang PY and Grossman J: Development of an Enhanced Recovery After Surgery (ERAS) approach for lumbar spinal fusion. J Neurosurg Spine 26: 411-418, 2017.

4. Fischer CR, Hanson G, Eller M and Lehman RA: A systematic review of treatment strategies for degenerative lumbar spine fusion surgery in patients with osteoporosis. Geriatr Orthop Surg Rehabil 7: 188-196, 2016.

5. Gazzeri R, Roperto R and Fiore C: Surgical treatment of degenerative and traumatic spinal diseases with expandable screws in patients with osteoporosis: 2-year follow-up clinical study. J Neurosurg Spine 25: 610-619, 2016.

6. Mueller JU, Baldauf J, Marx S, Kirsch M, Schroeder HW and Pillich DT: Cement leakage in pedicle screw augmentation: A prospective analysis of 98 patients and 474 augmented pedicle screws. J Neurosurg Spine 25: 103-109, 2016.

7. Pakzaban P: Modified mini-open transforaminal lumbar interbody fusion: Description of surgical technique and assessment of free-hand pedicle screw insertion. Spine (Phila Pa 1976) 41: E1124-E1130, 2016 
8. Cannestra AF, Peterson MD, Parker SR, Roush TF, Bundy JV, Turner AW: MIS expandable interbody spacers: A literature review and biomechanical comparison of an expandable MIS TLIF with conventional TLIF and ALIF. Spine 41 (Suppl 8): S44-S49, 2016.

9. Weiss HR and Moramarco M: Congenital scoliosis (mini-review). Curr Pediatr Rev 12: 43-47, 2016.

10. Mundis GM, Eastlack RK, Moazzaz P, Turner AW and Cornwall GB: Contribution of round vs. rectangular expandable cage endcaps to spinal stability in a cadaveric corpectomy model. Int J Spine Surg 9: 53, 2015.

11. Chen YL, Chen WC, Chou CW, Chen JW, Chang CM, Lai YS, Cheng CK and Wang ST: Biomechanical study of expandable pedicle screw fixation in severe osteoporotic bone comparing with conventional and cement-augmented pedicle screws. Med Eng Phys 36: 1416-1420, 2014.

12. Gonzalez-Blohm SA, Doulgeris JJ, Aghayev K, Lee WE III Laun J and Vrionis FD: In vitro evaluation of a lateral expandable cage and its comparison with a static device for lumbar interbody fusion: A biomechanical investigation. J Neurosurg Spine 20: 387-395, 2014

13. Bartanusz V, Harris J, Moldavsky M, Cai Y and Bucklen B: Short segment spinal instrumentation with index vertebra pedicle screw placement for pathologies involving the anterior and middle vertebral column is as effective as long segment stabilization with cage reconstruction: A biomechanical study. Spine 40: 1729-1736, 2015.

14. Mantell M, Cyriac M, Haines CM, Gudipally M and O'Brien JR Biomechanical analysis of an expandable lateral cage and a static transforaminal lumbar interbody fusion cage with posterior instrumentation in an in vitro spondylolisthesis model. J Neurosurg Spine 24: 32-38, 2016.

15. Xie W, Gao P and Ji L: Three-dimensional spiral CT measurement of atlantal pedicle and its clinical application. Exp Ther Med 14: $1467-1474,2017$
16. Zhou W, Kong W, Zhao B, Fu Y, Zhang T and Xu J: Posterior internal fixation plus vertebral bone implantation under navigational aid for thoracolumbar fracture treatment. Exp Ther Med 6: 152-158, 2013.

17. Donnelly DJ, Abd-El-Barr MM and Lu Y: Minimally invasive muscle sparing posterior-only approach for lumbar circumferential decompression and stabilization to treat spine metastasis - technical report. World Neurosurg 84: 1484-1490, 2015.

18. Demir T: A new alternative to expandable pedicle screws: Expandable poly-ether-ether-ketone shell. Proc Inst Mech Eng H 229: 386-394, 2015.

19. Kang SH, Cho YJ, Kim YB and Park SW: Pullout strength after expandable polymethylmethacrylate transpedicular screw augmentation for pedicle screw loosening. J Korean Neurosurg Soc 57: 229-234, 2015.

20. Lehman RA Jr, Kang DG and Wagner SC: Management of osteoporosis in spine surgery. J Am Acad Orthop Surg 23: 253-263, 2015.

21. Eschler A, Ender SA, Schiml K, Mittlmeier T and Gradl G: Bony healing of unstable thoracolumbar burst fractures in the elderly using percutaneously applied titanium mesh cages and a transpedicular fixation system with expandable screws. PLoS One 10: e0117122, 2015.

22. Galbusera F, Volkheimer D, Reitmaier S, Berger-Roscher N, Kienle A and Wilke HJ: Pedicle screw loosening: A clinically relevant complication? Eur Spine J 24: 1005-1016, 2015.

23. Jandial R, Kelly B and Chen MY: Posterior-only approach for lumbar vertebral column resection and expandable cage reconstruction for spinal metastases. J Neurosurg Spine 19: 27-33, 2013.

This work is licensed under a Creative Commons Attribution-NonCommercial-NoDerivatives 4.0 International (CC BY-NC-ND 4.0) License. 